(C) 2000 International Press

Adv. Theor. Math. Phys. 6 (2000) 1635-1655

\title{
Connectedness Of The
}

\section{Boundary In The AdS/CFT}

\section{Correspondence}

\author{
Edward Witten and S.-T. Yau
}

Department Of Physics, California Institute of Technology

Pasadena, CA 91106

and

Institute For Advanced Study, Olden Lane, Princeton, NJ 08540

Department of Mathematics, Harvard University

Cambridge, MA 02138

\begin{abstract}
Let $M$ be a complete Einstein manifold of negative curvature, and assume that (as in the AdS/CFT correspondence) it has a Penrose compactification with a conformal boundary $N$ of positive scalar curvature. We show that under these conditions, $H_{n}(M ; \mathbf{Z})=0$ and in particular $N$ must be connected. These results resolve some puzzles concerning the AdS/CFT correspondence.
\end{abstract}

e-print archive: http://xxx.lanl.gov/hep-th/9910245 


\section{Introduction}

Suppose that $M$ is a complete Einstein manifold of negative curvature and dimension $n+1$ and that the conformal boundary of $M$, in the sense of Penrose [1], is an $n$-manifold $N$. This means that $M$ is the interior of an $n+1$-dimensional manifold-with-boundary $\bar{M}$, whose boundary is $N$, and that the metric $g$ of $M$ can be written near the boundary as

$$
g=\frac{1}{t^{2}}\left(d t^{2}+g_{i j}(x, t) d x^{i} d x^{j}\right),
$$

where $t$ is a smooth function with a first order zero on $\partial \bar{M}$, and positive on $M$, and $g_{i j}(x, t) d x^{i} d x^{j}$ is an $t$-dependent family of metrics on $M$. Thus, $t \geq 0$ on $\bar{M}$ and $t=0$ on $N$. In this situation, $g_{0}=g(x, 0)$ is a metric on $N$. If $t$ is replaced by a different function with a first order zero on $\partial \bar{M}$, say $t^{\prime}=e^{\omega} t$, then $g_{0}$ undergoes a conformal transformation $g_{0} \rightarrow g_{0}^{\prime}=e^{2 \omega} g_{0}$, so $N$ actually has a natural conformal structure but not a natural metric. If, in the conformal class of metrics on $N$, there is a representative with positive (or zero, or negative) scalar curvature, then we say that $N$ has positive (or zero, or negative) scalar curvature.

There is a correspondence between conformal field theory on $N$ and quantum gravity, or string theory, on $M$ [2] - [4]. To be more precise, the correspondence asserts (see [4], section 3 ) that to do conformal field theory on $N$ with a given conformal structure $g_{0}$ on $N$, one must sum over contributions of all possible $n+1$-dimensional Einstein manifolds $M$ with conformal boundary $N$ and induced conformal structure $g_{0}$. Actually, the full correspondence involves a number of additional details that we will omit in the present paper. For example, one usually must consider not $n+1$-dimensional Einstein manifolds $M$, but manifolds of dimension $n+k+1$ obeying appropriate supergravity equations and asymptotic at infinity to $X=M \times Y$, where $Y$ is a fixed compact $k$-manifold characteristic of the conformal field theory that one chooses to consider. (Examples are given in [2].) Our results could possibly be extended to theorems about the possible $X$ 's (showing for example that under suitable hypotheses the ideal boundary of $X$ is connected), but for simplicity we will consider only the case that $X=M \times Y$, and analyze the possible $M$ 's.

\section{Topological Conditions}

Presented with this correspondence, one wonders how one can char- 
acterize the $M$ 's that exist for given $N$. Are there general topological conditions on $M$ ? For some choices of $N$, can one find all of the $M$ 's? In this paper, we will prove the following general restriction: if $N$ has positive scalar curvature (and is nonempty), then $H_{n}(M ; \mathbf{Z})=0$. We explain below the physical interpretation of this restriction.

The role of positive scalar curvature is suggested by the most canonical example of a complete, negatively curved Einstein manifold, namely hyperbolic space $\mathbf{H}^{n+1}$, or - as it is known in the supergravity literature - Anti de Sitter space $\mathrm{AdS}_{n+1}$. In this case, the conformal boundary $N$ is a sphere $\mathbf{S}^{n}$, with the standard conformal structure which has as a representative the standard "round" metric. This metric certainly has positive scalar curvature. Because of the basic role played by this example, the correspondence between quantum gravity in $n+1$ dimensions and conformal field theory in $n$ dimensions is sometimes called the AdS/CFT correspondence.

In general, conformal field theory can make sense on a manifold of negative scalar curvature, but the specific conformal field theories that arise in the AdS/CFT correspondence, at least in the examples studied so far, are well-behaved only when the scalar curvature of $N$ is non-negative. In the important case that $N$ is a four-manifold, this can be seen directly: in this case, the conformal field theories are fourdimensional gauge theories which contain scalar fields whose potential is unstable if the scalar curvature of $N$ is negative. More generally, one sees by considering the action of a suitable brane in $M$ [5], [6] that if $N$ has negative scalar curvature, the theory is unstable. The argument, whose details we recall in section 2 , is made by considering a brane in $M$ whose worldvolume is a codimension one hypersurface $\Sigma \subset M$. One considers the brane action $L(\Sigma)$ (which we will define in section 2) and shows that it is unbounded below if $N$ has negative scalar curvature. If $N$ has positive scalar curvature, the theory is stable, and if $N$ has zero scalar curvature, it may be stable or unstable depending on further details.

\section{Two Puzzles Concerning The AdS/CFT Correspondence}

We therefore limit ourselves to the case that $N$ has positive scalar curvature. Consider the following two puzzles concerning the AdS/CFT correspondence: 
(1) Can it happen that $N$ is not connected but is a union of disjoint components $N_{i}, i=1, \ldots, s$, each of positive scalar curvature? If so, the AdS/CFT correspondence does not make much sense. For conformal field theory on a union of disjoint manifolds $N=\cup_{i} N_{i}$ is just the product of the theories on the different $N_{i}$ 's. There is no evident way to couple them, and hence no candidate for how to interpret the contribution of an $M$ whose boundary is $\cup_{i} N_{i}$.

(2) For given $N$ of positive scalar curvature, can $M$ contain "wormholes"? A wormhole is obtained by cutting out two balls from a manifold $M_{0}$ and then gluing together their boundaries to make a new manifold $M$. If $M$ can have such wormholes, then to understand the conformal field theory on $N$, we will have to come to grips with the strange behavior of quantum gravity in the presence of wormholes [7], [8].

We will resolve both of these problems by showing that they simply do not arise for $N$ of positive scalar curvature. This follows from our result that for such $N, H_{n}(M ; \mathbf{Z})=0$. Indeed, in (1), if the number of boundary components is greater than one, then a hypersurface that is near one of the boundary components (defined by an equation such as $t=\epsilon$, where $t$ is the coordinate used in (1.1) and $t=0$ defines the boundary in question) is a nonzero element of $H_{n}(M ; \mathbf{Z})$. Hence vanishing of $H_{n}(M ; \mathbf{Z})$ implies that $N$ is connected. Likewise, in (2), a spacetime $M$ with wormholes would have nonzero $H_{n}(M ; \mathbf{Z})$, since the boundary of either of the balls removed from $M_{0}$ is a nonzero element of $H_{n}(M ; \mathbf{Z})$. Hence vanishing of $H_{n}(M ; \mathbf{Z})$ implies that there are "no wormholes."

Results of this type definitely depend on $N$ having positive scalar curvature. For example, let $Q$ be any compact negatively curved Einstein manifold of dimension $n$, with metric $g_{i j} d x^{i} d x^{j}$. Then a complete Einstein metric of negative curvature on $M=Q \times \mathbf{R}$ is given by the simple formula

$$
d s^{2}=d t^{2}+\cosh ^{2} t g_{i j} d x^{i} d x^{j}
$$

The conformal boundary of $M$ consists of two copies of $Q$, at $t=$ $\pm \infty$. This shows that if the conformal boundary of $M$ has negative scalar curvature, then $H_{n}(M ; \mathbf{Z})$ can be nonzero. Note that in this example, each component of $N$ has negative scalar curvature. In fact, 
our proof shows that $H_{n}(M ; \mathbf{Z})=0$ if any component of $N$ has positive scalar curvature.

An interesting corollary of the fact that, under the stated hypotheses, $N$ must be connected is that the natural map from $\pi_{1}(N)$ to $\pi_{1}(M)$ is surjective. (For example, $M$ must be simply connected if $N$ is simply connected.) Otherwise, by taking a suitable cover of $M$, one could make an example with disconnected $N$ of positive scalar curvature.

Structure Of The Argument

The proof that $H_{n}(M ; \mathbf{Z})=0$ for a boundary of positive scalar curvature will be made by showing, by a local calculation, that the brane action $L(\Sigma)$ cannot have a minimum, and also by showing, using nonlinear analysis, that there is a minimum in each nonzero homology class if the boundary has a component of positive scalar curvature. Combining these results, it follows that $H_{n}(M ; \mathbf{Z})=0$ if the boundary has such a component. The local computation is presented in section 2 and the global one in section 3 .

\section{Local Calculation}

Let $M$ be an $n+1$-dimensional Einstein manifold of negative curvature and nonempty conformal boundary, for some $n \geq 2$. Let $\Sigma$ be a compact hypersurface in $M$. We denote its area or volume as $A$. Since $M$ has a nonempty boundary, the volume form $\Theta$ of $M$ is exact, say $\Theta=d \Lambda$ for an $n$-form $\Lambda$. The brane action (for a BPS brane) is defined by

$$
L(\Sigma)=A-n \int_{\Sigma} \Lambda
$$

Note that if $\Sigma$ is the boundary of a domain $\Omega$, we have

$$
L(\Sigma)=A-n V
$$

where

$$
V=\int_{\Omega} \Theta
$$

is the volume enclosed by $\Sigma$. $\Lambda$ is not unique, but changing $\Lambda$ will add to $L$ a term that is a constant in each homology class, and this 
does not affect the variational problem that we will consider below. (In the physical application, $\Lambda$ is an $n$-form field of the appropriate supergravity theory, and any choice of $\Lambda$ makes sense.)

The importance of positive curvature for the boundary is that it is necessary in order to ensure that $L(\Sigma)$ is bounded below. This follows from a computation performed in [5] and in greater generality in [6]. As in the introduction, we write the metric near the boundary as

$$
d s^{2}=\frac{1}{t^{2}}\left(d t^{2}+g_{i j}(x, t) d x^{i} d x^{j}\right) .
$$

We consider $\Sigma$ to be homotopic to the boundary and to be defined by an equation $t=t\left(x^{i}\right)$. We write

$$
t= \begin{cases}2 \phi^{-2 /(n-2)} & \text { for } n>2 \\ 2 e^{-\phi} & \text { for } n=2 .\end{cases}
$$

The brane action then becomes for small $t$ or equivalently large $\phi$

$$
L=\left\{\begin{array}{cc}
\frac{1}{2^{n-3}(n-2)^{2}} \int_{\Sigma} d^{n} x \sqrt{g}\left(|d \phi|^{2}+\frac{n-2}{4(n-1)} \phi^{2} R\right. & \\
\left.+\mathcal{O}\left(\phi^{2(n-4) /(n-2)}\right)\right) & \text { for } n>2 \\
\frac{1}{2} \int_{\Sigma} d^{2} x \sqrt{g}\left(|d \phi|^{2}+\phi R+\mathcal{O}\left(e^{-2 \phi}\right)\right) & \text { for } n=2 .
\end{array}\right.
$$

Here we have identified $\Sigma$ with the boundary at $t=0$, and we regard $g_{i j}(x, 0)$ as a metric $g_{i j}$ on $\Sigma ; R$ is the scalar curvature of this metric. ${ }^{1}$ The brane action is conformally invariant; indeed, under $t \rightarrow e^{\omega} t$ (where $\omega$ is a function on the boundary, that is, on $\Sigma$ ), we have $g_{i j} \rightarrow e^{2 \omega} g_{i j}$ and in view of $(2.5)$

$$
\phi \rightarrow \begin{cases}\exp (-(n-2) \omega / 2) \phi & \text { for } n>2 \\ \phi-\omega & \text { for } n=2\end{cases}
$$

For $L$ to be bounded below, it must be bounded below in the region of large $\phi$, where the corrections in (2.6) can be dropped. Whether this is so depends, for $n>2$, on the spectrum of the conformally invariant operator

$$
\triangle^{\prime}=\triangle+\frac{n-2}{4(n-1)} R,
$$

\footnotetext{
${ }^{1}$ In deducing (2.6), one uses the Einstein equations to determine the behavior of $g_{i j}(x, t)$ near $t=0$. For details, see [6], eqns. (3.6)-(3.8).
} 
where $\triangle$ is the Laplacian. If $\triangle^{\prime}$ is positive definite, $L$ is bounded below at least in the region of large $\phi$; if it has a negative eigenvalue, then $L$ is unbounded below; and if the smallest eigenvalue of $\Delta^{\prime}$ is zero, then one must consider the correction terms in (2.6) to determine if $L$ is bounded below in the large $\phi$ region. The lowest eigenvalue of $\Delta^{\prime}$ is positive, negative, or zero depending on whether, in the conformal class of the metric $g_{i j}$ on $\Sigma$, there is a representative of positive, negative, or zero scalar curvature.

Thus we learn that, for $n>2$, stability requires that $\Sigma$ have nonnegative scalar curvature. For $n=2$, the same conclusion can be reached by first replacing $g$ with a conformally equivalent metric $e^{2 \omega} g$ with constant $R$, and then noting that for constant $\phi$ and $\phi \rightarrow+\infty, L$ is bounded below if $R \geq 0$ and unbounded below if $R<0$.

\section{Properties Of A Minimum of $L$}

So far we have merely summarized the considerations in [5], [6]. Now, assuming that the boundary of $M$ has positive scalar curvature, we want to try to get a restriction on the topology of $M$. The computation performed above suggests that (for a boundary of positive scalar curvature) $L$ is bounded below. If so, we may expect that $L$ will have an absolute minimum for each nonzero choice of the homology class of $\Sigma$ in $H_{n}(M ; \mathbf{Z})$. (The reason that one suspects a minimum for each homology class, not each homotopy class, is that in varying a hypersurface $\Sigma$ to minimize $L, \Sigma$ may develop a singularity. In passing through such a singularity, the homology class of $\Sigma$ does not change, but the homotopy class may.) The existence of such a minimum will be proved in section 3. We will now show, however, by a local computation that $L$ cannot have a minimum. Combining these results, it will follow that $H_{n}(M ; \mathbf{Z})=0$, which is the result that was promised in the introduction.

We assume that the Einstein manifold $M$ obeys

$$
R_{I J}=-n g_{I J} .
$$

(The choice of constant on the right hand side is correlated with the choice of constant $n$ multiplying the second term in the brane action (2.2). We denote indices of $M$ by $I, J, K=1, \ldots, n+1$ and indices of the hypersurface $\Sigma$ by $i, j, k=1, \ldots, n$.) 
We now want to study a hypothetical extremum $\Sigma$ of $L$. We suppose that $\Sigma$ is an embedded submanifold. In a neighborhood of $\Sigma$, we can pick one coordinate, $r$, to be the distance from $\Sigma$, and pick the other coordinates $x^{i}$ so that the metric near $\Sigma$ is

$$
d s^{2}=d r^{2}+\widehat{g}_{i j}(x, r) d x^{i} d x^{j}
$$

We let $g_{i j}=\widehat{g}_{i j}(x, 0)$ be the metric on $\Sigma$, and we write

$$
\begin{aligned}
& \dot{g}_{i j}=\left.\frac{\partial \widehat{g}_{i j}(x, r)}{\partial r}\right|_{r=0} \\
& \ddot{g}_{i j}=\left.\frac{\partial^{2} \widehat{g}_{i j}(x, r)}{\partial r^{2}}\right|_{r=0} .
\end{aligned}
$$

(Thus, the second fundamental form of $\Sigma$ is $\dot{g} / 2$, and the mean curvature is $\operatorname{Tr} g^{-1} \dot{g} / 2$.)

We can describe a fluctuation in the position of $\Sigma$ by specifying $r$ as a function of $x$. To determine the conditions for $\Sigma$ to be a local minimum of $L$, we need to evaluate $L$ up to second order in $r$. Writing $\widehat{g}=g+r \dot{g}+\frac{1}{2} r^{2} \ddot{g}+\ldots$, we calculate to this order

$$
\begin{aligned}
A= & \int d^{n} x \sqrt{\operatorname{det}\left(\widehat{g}_{i j}+\partial_{i} r \partial_{j} r\right)} \\
= & \int d^{n} x \sqrt{\operatorname{det} g}\left(1+\frac{r}{2} \operatorname{Tr} g^{-1} \dot{g}+\frac{r^{2}}{8}\left(\operatorname{Tr} g^{-1} \dot{g}\right)^{2}\right. \\
& \left.+\frac{r^{2}}{4} \operatorname{Tr} g^{-1} \ddot{g}-\frac{r^{2}}{4} \operatorname{Tr}\left(g^{-1} \dot{g} g^{-1} \dot{g}\right)+\frac{1}{2}|d r|^{2}\right) .
\end{aligned}
$$

Also, to this order

$$
n V=\text { const }+n \int d^{n} x \sqrt{g}\left(r+\frac{r^{2}}{4} \operatorname{Tr} g^{-1} \dot{g}\right) .
$$

So

$$
\begin{aligned}
L= & A-n V \\
= & \text { const }+\int d^{n} x \sqrt{g}\left(\frac{r}{2} \operatorname{Tr} g^{-1} \dot{g}-r n+\frac{r^{2}}{8}\left(\operatorname{Tr} g^{-1} \dot{g}\right)^{2}-\frac{n r^{2}}{4} \operatorname{Tr} g^{-1} \dot{g}\right. \\
& \left.+\frac{r^{2}}{4}\left(\operatorname{Tr} g^{-1} \ddot{g}-\operatorname{Tr}\left(g^{-1} \dot{g} g^{-1} \dot{g}\right)\right)+\frac{1}{2}|d r|^{2}\right) .
\end{aligned}
$$


The condition for $L$ to be stationary at $r=0$ is

$$
\operatorname{Tr} g^{-1} \dot{g}=2 n \text {. }
$$

For $r=0$ to be a local minimum requires

$$
\operatorname{Tr} g^{-1} \ddot{g} \geq \operatorname{Tr}\left(g^{-1} \dot{g} g^{-1} \dot{g}\right) .
$$

If $N$ is a real symmetric $n \times n$ matrix, then

$$
\operatorname{Tr} N^{2} \geq \frac{1}{n}(\operatorname{Tr} N)^{2}
$$

with equality only if and only if $N$ is a multiple of the identity. Applying this to $N=g^{-1} \dot{g}$, for which $\operatorname{Tr} N=2 n$, we learn that

$$
\operatorname{Tr}\left(g^{-1} \dot{g} g^{-1} \dot{g}\right) \geq 4 n,
$$

with equality precisely if $\dot{g}=2 g$.

Now we look at the Einstein equations. If $\Gamma$ are the ( $r$-dependent) Christoffel symbols of $\Sigma$ in the metric $g_{i j}(x, r) d x^{i} d x^{j}$, then the nonzero Christoffel symbols $\widehat{\Gamma}$ of $M$ in the metric (2.10) are

$$
\begin{aligned}
& \widehat{\Gamma}_{j k}^{i}=\Gamma_{j k}^{i} \\
& \widehat{\Gamma}_{j k}^{r}=-\frac{1}{2} \dot{g}_{j k} \\
& \widehat{\Gamma}_{r j}^{i}=\frac{1}{2} g^{i s} \dot{g}_{j s} .
\end{aligned}
$$

Let $R_{i j}$ and $R$ be the Ricci tensor and scalar of $\Sigma$, and $\widehat{R}_{i j}, \widehat{R}$ the analogous objects of $M$. The relevant part is

$$
\widehat{R}_{r r}=-\frac{1}{2} \operatorname{Tr} g^{-1} \ddot{g}+\frac{1}{4} \operatorname{Tr} g^{-1} \dot{g} g^{-1} \dot{g}
$$

Now we use the Einstein equations at $r=0$; the equation $\widehat{R}_{r r}=$ $-n g_{r r}=-n$ gives

$$
\frac{1}{2} \operatorname{Tr}\left(g^{-1} \ddot{g}\right)-\frac{1}{4} \operatorname{Tr}\left(g^{-1} \dot{g} g^{-1} \dot{g}\right)=n .
$$

Using also the inequality (2.16) that followed from stability, we get

$$
4 n \geq \operatorname{Tr}\left(g^{-1} \dot{g} g^{-1} \dot{g}\right) .
$$


Comparing to (2.18), we learn that all the inequalities must be equalities, forcing $\dot{g}=2 g, R=0$, and $\operatorname{Tr} g^{-1} \ddot{g}=4 n$.

So the possibility that the action $L$ has a stable nondegenerate minimum is excluded. Note that the analysis has been purely local and makes no assumption about the global structure of $M$.

As for the case of a degenerate minimum where the order $r^{2}$ term vanishes, a further analysis that we will explain momentarily shows that this can happen only in an example of the following type. If the metric $g_{i j}$ on $\Sigma$ is Ricci-flat, then the metric

$$
d s^{2}=d r^{2}+e^{2 r} g_{i j} d x^{i} d x^{j}
$$

on $\mathbf{R} \times \Sigma$ obeys the $n+1$-dimensional Einstein equations with cosmological constant. For any constant $c$, the submanifold $\Sigma_{c}$ of $\mathbf{R} \times \Sigma$ defined by $r=c$ is a stationary point of $L$. The action $L\left(\Sigma_{c}\right)$ is independent of $c$, so this is a degenerate critical point. Conformal infinity consists of a copy of $\Sigma$ at $r=\infty$, with zero scalar curvature. Thus this type of example is impossible if we assume that the boundary has positive scalar curvature. (In this type of example, there is also a sort of cusp at $r=-\infty$, so there is no Penrose compactification even with nonpositive curvature on the boundary.)

We conclude by giving the proof that a degenerate minimum of $L$ must be of the form just described. Let $L(c)=L\left(\Sigma_{c}\right)$. We have from $(2.14)$

$$
\frac{d L}{d c}=-\int_{\Sigma_{c}} d^{n} x \sqrt{g} F
$$

where

$$
F=n-\frac{1}{2} \operatorname{Tr} g^{-1} \dot{g}
$$

Since we assume that $L$ is locally minimized at $c=0$, we have $d L / d c \geq$ 0 for small positive $c$, and hence

$$
\int d^{n} x \sqrt{g} F \leq 0
$$

On the other hand,

$$
\frac{d}{d r} F=\frac{1}{2} \operatorname{Tr}\left(g^{-1} \dot{g} g^{-1} \dot{g}\right)-\frac{1}{2} \operatorname{Tr} g^{-1} \ddot{g} .
$$


Using (2.21), this becomes

$$
\frac{d F}{d r}=-n+\frac{1}{4} \operatorname{Tr}\left(g^{-1} \dot{g} g^{-1} \dot{g}\right)
$$

Using (2.17), with $N=g^{-1} \dot{g}$, this implies an inequality

$$
\frac{d F}{d r} \geq-\frac{1}{n} F(2 n-F)
$$

We have $F(0)=0$, since $\Sigma_{0}$ is a critical point of $L(\Sigma)$. For small positive $r, F$ has the same sign as $F^{\prime}$ since $F(r)=\int_{0}^{r} d t F^{\prime}(t)$. (2.29) implies that if $F(r)$ is negative for small positive $r$, then $d F / d r$ is positive. This is a contradiction, so $F \geq 0$ for small positive $r$.

Comparing to (2.26), we learn that $F$ is identically zero for all sufficiently small positive $r$. It follows from (2.24) that $L(c)$ is independent of $c$ for small positive $c$. As we deduced from (2.22), at any value of $c$ for which $d L / d c=d^{2} L / d c^{2}=0$, we have $d g_{i j} / d c=2 g_{i j}$. Hence

$$
g_{i j}(x, c)=e^{2 c} g_{i j}(x, 0)
$$

for sufficiently small positive $c$. By real analyticity (or the Einstein equations) this is true for all $c$, and the Einstein equations (2.9) also imply that $g_{i j}(x, 0)$ is a Ricci-flat metric on $\Sigma$. Thus, we have shown that a degenerate minimum of the functional $L(\Sigma)$ has the special form given in (2.23) and in particular cannot exist if the boundary has positive scalar curvature.

In the above, we can replace the Einstein equation $R_{I J}=-n g_{I J}$ by an inequality $R_{I J} \geq-n g_{I J}$, since this would only improve the crucial inequality (2.21). Physically, this corresponds to having additional matter fields excited in an asymptotically AdS spacetime.

We summarize our results as follows:

Theorem 2.1. The functional $L(\Sigma)=A-n \int_{\Sigma} \Lambda$ for an embedded hypersurface $\Sigma$ in an $n+1$-dimensional Einstein manifold $M$ of Ricci curvature greater than or equal to $-n$ does not have any local minimum. Any critical point of this functional is either unstable or is neutrally stable and of the form given in (2.23). The neutrally stable case is only possible if the Ricci curvature is precisely $-n$. 


\section{Existence}

In this section, we will prove existence theorems for a hypersurface $\Sigma$, in a given homology class, that minimizes the functional $L(\Sigma)=$ Area $(\Sigma)-n \int_{\Sigma} \Lambda$. Here $\Sigma$ is a hypersurface in a complete $n+1$ dimensional manifold $M$ of Ricci curvature $-n$ that has a conformal boundary as described in the introduction and the last section.

If instead $M$ were compact, and we have an upper bound on the area of $\Sigma$ and a lower bound on $L(\Sigma)$, then existence of a minimizing hypersurface $\Sigma$ in a given homology class in $M$ follows from very general grounds. In fact, any sequence of hypersurfaces of bounded area in a compact manifold has a convergent subsequence. For compact $M$, if we assume that $\Sigma$ is an embedded hypersurface, then both the upper bound on the area in a minimum of $L(\Sigma)$ and the lower bound on $L(\Sigma)$ follow from the fact that the potentially negative term $-n \int_{\Sigma} \Lambda$ in the definition of $L(\Sigma)$ is bounded below by minus the volume of $M$. So if we make $M$ compact by cutting off the "ends," then $\Sigma$ exists.

The $\Sigma$ obtained this way, as the limit of a sequence of embedded hypersurfaces $\Sigma_{i}$ chosen to minimize $\lim _{i \rightarrow \infty} L\left(\Sigma_{i}\right)$, might a priori have very bad singularities. However, rather deep results in geometric measure theory [9] - [12] show that such a limiting $\Sigma$ has singularities only in codimension $\geq 7$. (These results are obtained for area-minimizing hypersurfaces. The possible singular behavior at interior points of $M$ of a hypersurface minimizing $L=A-n \int \Lambda$ is the same as for areaminimizing hypersurfaces, since the second term is less important near a singularity.) Existence of a minimizing hypersurface $\Sigma$ that is smooth except in high codimension is good enough for our purposes, because the arguments of section 2, though formulated for smooth $\Sigma$, can be extended to the case that $\Sigma$ has a singularity of high codimension. ${ }^{2}$

To apply this existence result for $\Sigma$ to the case that $M$ is noncompact and has a Penrose compactification, we first introduce a cutoff in the volume of $M$ as follows. We recall that near each conformal

\footnotetext{
${ }^{2}$ Curiously, in supersymmetric examples of the AdS/CFT corresponce (and in fact, in all known examples) the dimension of $M$ is at most seven and hence the dimension of $\Sigma$ is at most six. So in the known applications, $\Sigma$ is always smooth.
} 
boundary component $\partial M_{i}$ of $M$, the metric of $\Sigma$ looks like

$$
\frac{1}{t^{2}}\left(d t^{2}+g_{i j}(x, t) d x^{i} d x^{j}\right)
$$

with the boundary being at $t=0$. We cut off the "ends" by restricting to $t \geq \epsilon_{i}$, with $\epsilon_{i}$ a small positive function on $\partial M_{i}$. With $M$ made compact in this way, existence of $\Sigma$ follows by the argument above. The main technical step in the present section is to prove, under certain conditions, that if the $\epsilon_{i}$ are sufficiently small, then the minimizer $\Sigma$ (or at least one of its components) does not intersect the boundary of $M$. Once this is known, a comparison with Theorem 2.1 will give our restrictions on the topology of $M$.

We turn now to the proofs. We begin with some preparations. The following is a well-known fact:

Lemma 1. Let $d$ be the geodesic distance function from a point $x_{0}$ in a manifold $M$ whose sectional curvature has an upper bound given by $k>0$. At points where $\sqrt{k} d<\pi / 2$ and $d$ is smooth, the second derivative (the Hessian) of $d$, in directions orthogonal to the tangent vector of the shortest geodesic joined to $x_{0}$, is not less than

$$
\frac{1}{d} \frac{\sqrt{k} d}{\tan \sqrt{k} d}
$$

Proof. Let $\sigma(s, t):(-a, a) \times[0, l] \rightarrow M$ be a family of geodesics so that

$$
\sigma(s, 0)=x_{0} \quad \text { for all } s,
$$

and at $s=0, \frac{d}{d s} \sigma(s, l)$ is perpendicular to $\frac{d \sigma}{d t}(0, l)$. We also suppose that $\sigma(s, l)$ is a geodesic. We shall parametrize $\sigma(0, t)$ by arc length so that $t=$ length $\sigma(0, t)$. Then $J(t)=\left.\frac{d}{d s} \sigma(s, t)\right|_{s=0}$ is perpendicular to $\frac{d \sigma}{d t}$ for all $t$. Direct calculation shows that

$$
\frac{d^{2}}{d s^{2}} \text { length }\left.\sigma(s, t)\right|_{s=0}=\int_{0}^{l}\left[\left|\frac{d}{d t} J\right|^{2}-\int_{0}^{l} R_{i j k l} J^{i} J^{k} \frac{d \sigma^{j}}{d t} \frac{d \sigma^{l}}{d t}\right] d t \text {. }
$$

Assume the sectional curvature $\Sigma R_{i j k l} X^{i} X^{k} Y^{j} Y^{l}$ to be less than $k\|X\|^{2}\|Y\|^{2}$ when $X \perp Y$. Then we see that

$$
\frac{d^{2}}{d s^{2}} \text { length }\left.(\sigma)\right|_{s=0} \geq \int_{0}^{l}\left\|\frac{d J}{d t}\right\|^{2}-k \int_{0}^{l}\|J\|^{2} .
$$


Assuming $\sqrt{k} l \leq \frac{\pi}{2}$, the right hand side is minimized by setting

$$
J(t)=\frac{\sin (\sqrt{k} t)}{\sin (\sqrt{k} l)} J(1) .
$$

Hence

$$
\frac{d^{2}}{d s^{2}} \text { length }\left.(\sigma)\right|_{s=0} \geq \frac{\sqrt{k}}{\tan (\sqrt{k} l)}
$$

Let $\rho=d^{2}$. Then the second derivative of $\rho$ is not less than

$$
\frac{2 \sqrt{k} d}{\tan (\sqrt{k} d)}
$$

if the derivatives are taken along directions orthogonal to the shortest geodesic joint to $x_{0}$. It is not less than 2 if the direction is tangential to the shortest geodesic joint to $x_{0}$. Hence the second derivative is always not less than

$$
f(\rho)=2 \min \left(\frac{\sqrt{k} d}{\tan (\sqrt{k} d)}, 1\right) .
$$

Let $\Sigma$ be a hypersurface with mean curvature bounded above by $c .^{3}$ Then restricting $\rho$ to $\Sigma$, we find

$$
\Delta_{\Sigma} \rho \geq n f(\rho)-2 c \sqrt{\rho}
$$

This follows because the second derivatives on $\Sigma$ differ from the ones on $\Sigma$ by the second fundamental form after being renormalized by the gradient of $\rho$, whose norm is less than $2 d=2 \sqrt{\rho}$. (The first derivative of the geodesic distance is not greater than one.)

Let us now assume that distance from $x \in \Sigma$ to $\partial M$ is greater than $\sqrt{R}$. Integrating the above inequality on $\Sigma \cap\{\rho \leq R\}$, we obtain

$$
2 \sqrt{R} \text { Area }[\Sigma \cap\{\rho=R\}] \geq \int(n f(\rho)-2 c \sqrt{\rho}) .
$$

(Here we have used the fact that, since the normal derivative of $d$ is at most 1 , the normal derivative of $\rho$ is not greater than $2 \sqrt{R}$ when $\rho=R$.)

\footnotetext{
${ }^{3}$ In the notation of section 2 , the mean curvature is $\operatorname{Tr} g^{-1} \dot{g} / 2$.
} 
Let $F(R)=$ Volume $[\Sigma \cap\{\rho \leq R\}]$. Then

$$
\begin{aligned}
\frac{d F}{d R} & \geq \frac{1}{2 \sqrt{R}} \text { Area }[\Sigma \cap\{\rho=R\}] \\
& \geq \int_{\rho \leq R}\left(\frac{n f(t)-2 c \sqrt{t}}{4 R}\right) \\
& \geq \inf _{t \leq R}\left(\frac{n f(t)-2 c \sqrt{t}}{4 R}\right) F(R) .
\end{aligned}
$$

In particular,

$$
(\log F(R))^{\prime} \geq(\log H(R))^{\prime}
$$

where

$$
H(R)=R^{n / 2} \exp \left\{\int_{0}^{R}\left(\inf _{s \leq t}\left(\frac{n f(s)-2 c \sqrt{s}}{4 t}\right)-\frac{n}{2 t}\right)\right\} .
$$

When $R \rightarrow 0, H(R) \rightarrow R^{n / 2}$ and $F(R) \sim C R^{n / 2}$. Hence

$$
F(R) \geq C R^{n / 2} \exp \left\{\int_{0}^{R}\left(\inf _{t \leq R}\left(\frac{n f(t)-2 c \sqrt{t}}{4 R}\right)-\frac{n}{2 t}\right)\right\} .
$$

Lemma 2. Let $\Sigma$ be a hypersurface with a mean curvature bounded above by $c$ in a manifold $M$ whose sectional curvature is bounded above by a constant $k$. Let $x_{0} \in \Sigma$ be a point so that the distance from $x_{0}$ to $\partial M$ is greater than $R$. Then if $R<\frac{\pi}{2 \sqrt{k}}$ and if the geodesic distance (of $M)$ from $x_{0}$ is smooth within $B_{x_{0}}(R)$ the ball of radius $R$, then the area of $B_{x_{0}}(R) \cap \Sigma$ is not less than $\widetilde{c} R^{n}$ where $\widetilde{c}$ depends only on $\operatorname{dim} M$, $\sqrt{k} R$ and $c R$.

The assumption that the geodesic function from $x_{0}$ is smooth will be true if $R$ is small enough. This can be seen as follows. The only reason that the geodesic distance may not be smooth at $x$ is that there may be two distinct geodesics with shortest distance joining $x$ to $x_{0}$. By minimizing the distance of such geodesics, one can find a point $x$ so that the distinct geodesics at $x$ have exactly opposite directions and hence there is a smooth geodesic loop at $x_{0}$.

Let us now assume that for some constant $\alpha>0$, the map $\pi_{1}\left(B_{x_{0}}(\alpha R)\right) \rightarrow \pi_{1}\left(B_{x_{0}}(R)\right)$ is trivial. Since $R<\frac{\pi}{2 \sqrt{k}}$, the exponential map from the tangent space at $x_{0}$ is non-singular everywhere in a ball of radius $R$. 
The geodesic loop at $x_{0}$ bounds a disk within $B_{x_{0}}(R)$ and hence can all be lifted up to the tangent space. This is not possible as the lifting of any geodesic at $x_{0}$ must be a straight line and cannot be a closed loop.

The condition for smoothness of geodesic distance is therefore satisfied if we can find $\alpha>0$ so that $\pi_{1}\left(B_{x_{0}}(\alpha R)\right) \rightarrow \pi_{1}\left(B_{x_{0}}(R)\right)$ is trivial for all $0<R<\frac{\pi}{2 \sqrt{k}}$ and for all $x_{0}$ with distance greater than $R$ from $\partial M$. This condition is clear for manifolds with compactification of the type described in section 1 .

From now on, we shall choose $R$ so that all the above assumptions are satisfied.

Suppose $\left\{x_{1}, x_{2}, \ldots, x_{m}\right\}$ are points on $\Sigma$ so that the balls $B_{x_{i}}\left(\frac{R}{2}\right)$ are mutually disjoint and $\Sigma \subset \cap_{i} B_{x_{i}}(R)$. We also assume distances from $x_{i}$ to $\partial M$ is greater than $\frac{R}{2}$. Then Area $(\Sigma) \geq \sum_{i}$ Area $\left(B_{x_{i}}\left(\frac{R}{2}\right) \cap \Sigma\right)$. Since each Area $\left(B_{x_{i}}\left(\frac{R}{2}\right) \cap \Sigma\right)$ is bounded from below by a positive constant depending only on $R, c$ and $\sqrt{k}$, we conclude that $m$ is dominated by Area $(\Sigma)$. This number $m$ can be considered as a quantity that measures the outer diameter of $\Sigma$.

Let us now assume that $M$ is a compact manifold with boundary components $\partial M_{1}, \ldots, \partial M_{k}$. We assume $k>1$. We consider domains $\Omega_{\Sigma}$ with boundary components given by $\partial M_{2}, \ldots, \partial M_{k}$ and an embedded hypersurface $\Sigma$ which is (compactly) homologous to $\partial M_{1}$. Then

$$
\begin{aligned}
L(\Sigma) & =\operatorname{Area}(\Sigma)-n \int_{\Sigma} \Lambda \\
& =\operatorname{Area}(\Sigma)+\sum_{i} n \int_{\partial M_{i}} \Lambda-n \operatorname{Vol}\left(\Omega_{\Sigma}\right) .
\end{aligned}
$$

If $\Sigma_{0}$ minimizes $L(\Sigma)$,

$$
\operatorname{Area}\left(\Sigma_{0}\right)-n \operatorname{Vol}\left(\Omega_{\Sigma_{0}}\right) \leq \operatorname{Area}\left(\partial M_{1}\right)-n \operatorname{Vol}(M)
$$

Assume that $\Sigma_{0}$ can be written as $\Sigma_{1}+\cdots+\Sigma_{k}$. Then either one of $\Sigma_{i}$ is in the interior of $M$ or all of them. intersect $\partial M$. In case all of them intersect $\partial M$, we argue as follows. We consider first the case that all of the $\Sigma_{i}$ intersect one of the $\partial M_{j}$ with $j>1$. From the above 
inequality (3.15), we know

$$
\sum_{i} \operatorname{Area}\left(\Sigma_{i}\right) \leq \operatorname{Area}\left(\partial M_{1}\right)
$$

By Lemma 2, the diameter of each $\Sigma_{i}$ is dominated by Area $\left(\Sigma_{i}\right)$ and hence by $a$ Area $\left(\partial M_{1}\right)$ where $a$ depends only on the upper bound of the sectional curvature of $M$ and the lower bound of the injectivity radius of $M$. (The mean curvature of each $\Sigma_{i}$ is $n$, according to (2.15).)

If $\Omega$ is the complement (in $M$ ) of the neighborhood of $\cup_{j>1}\left(\partial M_{j}\right)$ with radius of $a$ Area $\left(\partial M_{1}\right)$, then (as we are assuming that each $\Sigma_{i}$ intersects one of the $\partial M_{j}$ with $\left.j>1\right) \Omega \subset\left(M \backslash \Omega_{\Sigma_{0}}\right)$ and (3.15) shows

$$
\sum_{i \geq 1} \operatorname{Area}\left(\Sigma_{i}\right) \leq \operatorname{Area}\left(\partial M_{1}\right)-n \operatorname{Vol}(\Omega)
$$

In particular, Area $\left(\partial M_{1}\right)+n \operatorname{Vol}(M \backslash \Omega) \geq n \operatorname{Vol}(M)$.

We have then proved the following lemma:

Lemma 3. On a compact manifold $M$ with boundary $\partial M_{1}, \partial M_{2}, \ldots$, $\partial M_{k}$, let $\Sigma_{1}+\cdots+\Sigma_{i}$ be a sum of embedded cycles that minimizes the functional $L(\Sigma)=$ Area $(\Sigma)-n \operatorname{Vol} \Omega\left(\Sigma, \partial M_{2}, \ldots, \partial M_{k}\right)$, on the homology class of $\partial M_{1}$. Then one of the $\Sigma_{i}$ 's does not intersect $\partial M_{2} \cup$ $\cdots \cup \partial M_{k}$ if

$$
n \operatorname{Vol} M>\operatorname{Area}\left(\partial M_{1}\right)+n \operatorname{Vol} B_{d}\left(\partial M_{2} \cup \cdots \cup \partial M_{k}\right)
$$

where $d=a$ Area $\left(\partial M_{1}\right), B_{d}$ is the ball of radius $d$ around $\partial M_{2} \cup \cdots \cup$ $\partial M_{k}$ and $a$ depends only on the upper bound of the sectional curvature of $M$ and the lower bound of its injectivity radius.

In the case that $M$ is obtained from a manifold with a Penrose compactification by "cutting off" the ends by $t \geq \epsilon_{i}$ for $i \geq 2$, the inequality in Lemma 3 is obeyed if the $\epsilon_{i}$ are sufficiently small.

We still need a condition to ensure that a component of $\Sigma$ does not meet $\partial M_{1}$. When $\partial M_{1}$ is a conformal boundary at infinity, the computation at the beginning of section 2 suggests that $\Sigma$ will not be near $\partial M_{1}$ if $\partial M_{1}$ has positive scalar curvature; we want to make this 
more precise. In the present discussion, we have cut off the ends of $M$ and $\partial M_{1}$ is an ordinary boundary; positive scalar curvature at conformal infinity implies that (when $\partial M_{1}$ is sufficiently close to infinity) the mean curvature of $\partial M_{1}$ is greater than $n$.

Thus in general the assumption we want is that $\partial M_{1}$ has mean curvature greater than $n$. There is a foliation in a neighborhood of $\partial M_{1}$ so that the leaves are given by level sets of the distance function to $\partial M_{1}$. The mean curvature of the nearby level sets is still greater than $n$. The (outer) normal of these level sets defines a vector field $v$ in a neghborhood of $\partial M_{1}$ whose divergence is given by the mean curvature of the level sets. Hence

$$
\operatorname{div} v>n .
$$

Now if $\Sigma_{i}$ intersects $\partial M_{1}$, we can replace $\Sigma_{i}$ by intersecting it with the level sets and obtain a new surface $\widetilde{\Sigma}_{i}$. By applying the divergence theorem $(\operatorname{as} \operatorname{norm}(v)=1)$ to the domain $\Omega$ bounded by the difference of $\Sigma_{i}$ and $\widetilde{\Sigma_{i}}$, we get

$$
\text { Area } \begin{aligned}
\widetilde{\Sigma}_{i} & \leq \operatorname{Area} \Sigma_{i}-\int_{\Omega} \operatorname{div} v \\
& <\operatorname{Area} \Sigma_{i}-n \operatorname{Vol}(\Omega) .
\end{aligned}
$$

From this inequality, it is clear that $L\left(\widetilde{\Sigma_{i}}\right)<L\left(\Sigma_{i}\right)$. Hence $\Sigma_{i}$ cannot be part of the minimum of the functional $L$. In conclusion, if $\partial M_{1}$ has mean curvature strictly greater than $n$, it does not intersect any of the $\Sigma_{i}$, and hence under the hypotheses of Lemma 3, there must be a component $\Sigma_{i}$ which does not intersect any boundary components $\partial M_{j}$. We can deduce the following:

Lemma 4. Let $M$ be a compact manifold with boundary components $\partial M_{1}, \ldots, \partial M_{k}$. Assume that $\partial M_{1}$ has mean curvature greater than $n$. Let $B_{d}\left(\partial M_{2}, \ldots, \partial M_{k}\right)$ be a neighborhood of $\partial M_{2} \cup \cdots \cup \partial M_{k}$ with radius $d=a$ Area $\left(\partial M_{1}\right)$, where a depends only on the upper bound of sectional curvature of $M$ and the lower bound of the injectivity radius of $M$. Assume that

$$
n \operatorname{Vol}\left[M \backslash B_{d}\left(\partial M_{2}, \ldots, \partial M_{k}\right)\right]>\operatorname{Area}\left(\partial M_{1}\right) .
$$

Then when we minimize the functional $L(\Sigma)=\operatorname{Area}(\Sigma)-n \int_{\Sigma} \Lambda$ among embedded surfaces homologous to $\Sigma$ which bound a domain with 
$\partial M_{2} \cup \cdots \cup \partial M_{k}$, there must be a component of $\Sigma$ which does not touch $\partial M_{1} \cup \cdots \cup \partial M_{k}$. The singular set of $\Sigma$ is a closed set with at least codimension seven Hausdorff dimension.

This last statement follows from regularity theorems of geometric measure theory. The fact that the singular set has large Hausdorff codimension allows us to use arguments of section two. What one needs is to introduce in the computations in section 2 a cut off function $\varphi$ which is zero on the singular set and one outside an $\varepsilon$-neighborhood of the set. The contribution of $\nabla \varphi$ is $\frac{1}{\varepsilon}$. But the singular set has small measure and the integral of $|\nabla \varphi|^{2}$ tends to zero when $\varepsilon \rightarrow 0$.

If the number of $\partial M_{j}$ is greater than one, so that $\partial M_{1}$ is not homologous to zero, then a minimum of $L(\Sigma)$ exists in the homology class of $\partial M_{1}$ (as explained at the beginning of this section) and Lemma 4 gives a condition in which the minimum has a component that does not intersect the boundary of $\Sigma$. But the existence of such a component contradicts Theorem 2.1 if the Ricci curvature of $M$ is not less than $-n$. So we conclude:

Theorem 3.1. Let $M^{n+1}$ be a compact manifold with Ricci curvature not less than $-n$. Let $\partial M_{1}$ be one of the boundary components of $M$ so that $R_{\partial M_{1}}-R_{M}>\frac{1}{2} n(n+1)$ along $\partial M_{1}$. Assume that

$$
n \operatorname{Vol}\left[M \backslash B_{d}\left(\partial M_{2}, \ldots, \partial M_{k}\right)\right]>\operatorname{Area}\left(\partial M_{1}\right),
$$

where $d$ depends only on the upper bound of the sectional curvature of $M$ and the lower bound of the injectivity radius. $\left[B_{d}\left(\partial M_{2}, \ldots, \partial M_{k}\right)\right.$ is the neighborhood of radius d around the components of $\partial M \backslash \partial M_{1}$.] Then $\partial M$ has only one boundary component.

The above inequality on the volume is true if the boundary components $\partial M_{i}, i \geq 2$, are far away from $\partial M_{1}$. If $M$ is obtained by cutting off the "ends" in a Penrose compactification, we can obey this inequality by moving the $\partial M_{i}, i \geq 2$, close enough to infinity.

In the statement of Theorem 3.1, the condition $R_{\partial M_{1}}-R_{M}>\frac{1}{2} n(n+$ 1 ) ensures that the mean curvature of the boundary is greater than $n$.

Hence we have: 
Theorem 3.2. Let $M^{n+1}$ be a complete manifold without boundary with Ricci curvature not less than $-n$ and with a Penrose compactification such that at least one component of the conformal boundary of $M^{n+1}$ has positive scalar curvature. Then the conformal boundary of $M^{n+1}$ is connected. More generally, let $M^{n+1}$ be any complete manifold with curvature bounded from above and with Ricci curvature not less than $-n$. Suppose $\partial M$ is compact and $R_{\partial M}-R_{M}>\frac{1}{2} n(n+1)$ along $\partial M$. If the injectivity radius of $M$ is bounded from below by a positive constant, then $M$ is compact, with connected boundary $\partial M$.

Proof. If $M$ is not compact, we can exhaust $M$ by subdomains whose boundary is $\partial M$ and $\partial M_{2}, \ldots, \partial M_{k}$. We can make $\left(M \backslash B_{d}\left(\partial M_{2}, \ldots\right.\right.$, $\left.\partial M_{k}\right)$ ) to be arbitrarily large because $M$ is complete and noncompact and we can put an arbitrarily large number of balls $B_{x_{i}}(R)$ in $M \backslash B_{d}\left(\partial M_{2}, \ldots, \partial M_{k}\right)$ as long as the subdomain is large. Hence, we can obey the inequality in Lemma 4.

Theorem 3.3. Let $M$ be as in Theorem 3.2. Then the natural map $\pi_{1}(\partial M) \rightarrow \pi_{1}(M)$ is surjective.

Proof. Otherwise a non-trivial element of $\pi_{1}(M) \backslash \pi_{1}(\partial M)$ exists. We can form a covering manifold $\widetilde{M}$ of $M$ making use of this element. This $\widetilde{M}$ would have disconnected components.

Theorem 3.4. Let $M$ be as in Theorem 3.2. Then $H_{n}(M ; \mathbf{Z})$ is zero.

Proof. Let $\Sigma_{0}$ be a fixed embedded cycle representing an element in $H_{n}(M ; \mathbf{Z})$. Then we can study the functional $L(\Sigma)$ among embedded hypersurfaces $\Sigma$ homologous to $\Sigma_{0}$. In this case, $\Sigma \cup \Sigma_{0}$ is the boundary of a domain with components counted with multiplicity plus or minus one (according to the orientations). We can then apply Stokes's theorem on each subdomain to conclude that $\left|\int_{\Sigma} \Lambda\right|$ is bounded by Vol $M$ and $\int_{\Sigma_{0}}|\Lambda|$. The rest of the proof is the same as before.

We thank A. Strominger for discussions. Research of E.W. has been supported in part by NSF Grant PHY-95-13835 and the Caltech Discovery Fund. Research of S.-T. Yau supported in part by NSF Grant DMS-9803347 and DOE Grant FG02-88ER25065. 


\section{References}

[1] R. Penrose and W. Rindler, Spinors And Spacetime, Vol. 2, chapter 9 (Cambridge University Press, Cambridge, 1986).

[2] J. Maldacena, The Large $N$ Limit Of Superconformal Field Theories And Supergravity, hep-th/9711200.

[3] S.S. Gubser, I.R. Klebanov, and A.M. Polyakov, -Gauge Theory Correlators From Non-Critical String Theory, hep-th/9802109.

[4] E. Witten, Anti de Sitter Space And Holography, hep-th/9802150.

[5] J. Maldacena, J. Michelson, and A. Strominger, Anti de Sitter Fragmentation, JHEP 9902:011, 1999, hep-th/9804085.

[6] N. Seiberg and E. Witten, The D1/D5 System And Singular CFT, JHEP 9904:017, 1999, hep-th/9903224.

[7] S. Coleman, Why There Is Nothing Rather Than Something: A Theory Of The Cosmological Constant, Nucl. Phys., B310 (1988), 643.

[8] T. Banks, Prolegomena To A Theory Of Bifurcating Universes: A Nonlocal Solution To The Cosmological Constant Problem, Or Little Lambda Goes Back To The Future, Nucl. Phys., B309 (1988), 493.

[9] E. Gonzalez, U. Massari, and I. Tamanimi, On The Regularity Of Boundaries Of Sets Minimizing Perimeter With A Volume Constraint, Indiana U. Math. J., 32 (1983), 25.

[10] M. Grüter, Boundary Regularity For Solutions Of A Partitioning Problem, Arch. Rat. Mech. Anal., 97 (1987), 261.

[11] L. Simon, Lectures On Geometric Measure Theory, Proc. Center for Math. Anal., Australian Nat. Univ., 3 (1983).

[12] P. Sternberg and K. Zumbrun, On The Connectivity Of Boundaries Of Sets Minimizing Perimeter Subject To A Volume Constraint, Comm. Anal. Geom., 7 (1999), 199. 